\title{
Microscopic polyangiitis secondary to Mycobacterium abscessus in a patient with bronchiectasis: a case report
}

\author{
C. Addy ${ }^{1,2^{*}} \mathbb{D}$, G. Doran², A. L. Jones ${ }^{3}$, G. Wright ${ }^{4}$, S. Caskey ${ }^{2}$ and D. G. Downey ${ }^{1,2}$
}

\begin{abstract}
Background: Non-Tuberculous Mycobacterial-pulmonary disease (NTM-PD) is increasing in incidence and prevalence. Mycobacterium abscessus (M.abscessus) is a rapid growing multi-resistant NTM associated with severe NTM-PD requiring prolonged antibiotic therapy. Complications of therapy are common but reports on direct complications of active NTM-PD are rare. Vasculitis has been described as a rare complication of NTMPD, most often in individuals with inherited immune defects. This case is the first to describe an ANCA positive vasculitide (Microscopic Polyangiitis) secondary to M.abscessus pulmonary disease.

Case presentation: A 70 year old female with bronchiectasis underwent a clinical decline associated with the growth of M.abscessus and was diagnosed with NTM-PD. Before treatment could be initiated she developed small joint arthralgia and a glove and stocking axonal loss sensorimotor neuropathy. Positive Perinuclear Anti-Neutrophil Cytoplasmic Antibodies (P-ANCA) and Myeloperoxidase-ANCA (MPO-ANCA) titres led to a diagnosis of microscopic polyangiitis. Further investigation revealed reduced interferon-gamma production but no other significant immune dysfunction. Dual treatment with immunosuppressive therapy (Corticosteroids/Cyclophosphamide) for vasculitis and antimicrobial therapy for M.abscessus NTM-PD was initiated. Clinical stability was difficult to achieve with reductions in immunosuppression triggering vasculitic flares. One flare led to retinal vein occlusion with impending visual loss requiring escalation in immunosuppression to Rituximab infusions. An increase in immunosuppression led to a deterioration in NTM-PD necessitating alterations to antibiotic regimes. Adverse effects including alopecia and Achilles tendonitis have further limited antibiotic choices resulting in a strategy of pulsed intra-venous therapy to stabilise NTM-PD.

Conclusions: This is the first reported case of an ANCA positive vasculitis secondary to M.abscessus pulmonary disease. This rare but important complication had a significant impact on the patient adding to the complexity of an already significant disease and treatment burden. The potential role of reduced interferon-gamma production in this case highlights the importance of investigating immune function in those with mycobacterial infection and the intricate relationship between mycobacterial infection and immune dysfunction. Immune dysfunction caused by genetic defects or immunosuppressive therapy is a known risk factor for NTM-PD. Balancing immunosuppressive therapy with prolonged antimicrobial treatment is challenging and likely to become more common as the number of individuals being treated with biologics and immunosuppressive agents increases.
\end{abstract}

Keywords: Non-tuberculous mycobacteria, Non-tuberculous mycobacterial pulmonary disease Mycobacterium abscessus, Vasculitis, Interferon-gamma, Bronchiectasis

\footnotetext{
* Correspondence: c.addy@qub.ac.uk

${ }^{1}$ Centre for Experimental Medicine, Queen's University Belfast, 97 Lisburn

Road, Belfast BT9 7BL, Northern Ireland

${ }^{2}$ Regional Respiratory Centre, Belfast City Hospital, 51 Lisburn Road, Belfast

BT9 7AB, Northern Ireland

Full list of author information is available at the end of the article
}

(c) The Author(s). 2018 Open Access This article is distributed under the terms of the Creative Commons Attribution 4.0 International License (http://creativecommons.org/licenses/by/4.0/), which permits unrestricted use, distribution, and

reproduction in any medium, provided you give appropriate credit to the original author(s) and the source, provide a link to the Creative Commons license, and indicate if changes were made. The Creative Commons Public Domain Dedication waiver (http://creativecommons.org/publicdomain/zero/1.0/) applies to the data made available in this article, unless otherwise stated. 


\section{Background}

The incidence and prevalence of Non-Tuberculous Mycobacterial-pulmonary disease (NTM-PD) is increasing $[1,2]$. Understanding the spectrum of disease and pathogenicity of differing Non-Tuberculous Mycobacteria (NTM) has advanced in line with developments in microbiological detection highlighting the need to sub-speciate NTM and alter management accordingly [1]. NTM can cause progressive lung disease or reside within the lungs asymptomatically [1]. Treatment regimens are prolonged, toxic and difficult to tolerate. Decisions to commence treatment are based on a number of factors including disease severity, frequency of positive cultures, progression of radiological appearances, underlying lung disease, co-morbidities and pathogenicity of individual species [1].

Guidance on treating NTM-PD was first published in 2007 [3] with no further guidance published until the 2016 Cystic Fibrosis specific consensus document [4] and 2017 British Thoracic Society guidelines [5]. This is due to lack of evidence in this area and to the complexity of the disease. Increasing incidence and prevalence of NTM-PD highlights the need for further research and guidance [1].

Mycobacterium abscessus (M.abscessus) is one of the most pathogenic NTMs, particularly in those with underlying lung disease [5]. It is a multi-resistant organism with limited antibiotic options and is challenging to treat [5]. M. abscessus is a rapid growing NTM which can be sub-speciated into M.a.abscessus, M.a.massiliense and M.a.bolletii [5]. The initial treatment aim is eradication, which commonly fails and therefore long-term chronic suppressive therapy becomes necessary. Development of macrolide resistance is associated with reduced rates of culture conversion and increased chronic infection [5]. Evidence on optimal antibiotic regimes and longer-term outcomes of chronic suppressive treatment are limited $[3,5]$. Those with the subspecies M.a.abscessus have much lower rates of culture conversion [5]. The impact of M.abscessus infection on patients should not be underestimated because of prolonged toxic treatment regimens that it requires, the need for stringent infection control precautions and it's relative contra-indication to lung transplantation $[4,5]$.

\section{Case presentation}

A 70-year-old female had been symptomatic with a persistent non-productive cough and recurrent chest infections for 10 years. At presentation to clinic a CT thorax showed right middle lobe bronchiectasis. She was a life-long non-smoker with no childhood history of respiratory disease and normal baseline investigations for immune deficiency. Clinical stability was achieved with long term Azithromycin and regular airway clearance.
Staphyloccocus aureus (S.aureus) was cultured intermittently from sputum samples with repeatedly negative mycobacterial cultures. A repeat CT 6 years after her initial scan demonstrated progression of disease with bi-apical scarring, right middle lobe atelectasis, right upper lobe cylindrical bronchiectasis and reticulo-nodular densities in both lower lobes. (Fig. 1) Nebulised Tobramycin was trialled to suppress S.aureus and stabilise radiological appearances but stopped after 15 months due to worsening cough.

M. abscessus was first isolated from her sputum in November 2013; sub-speciated into M.a. abscessus. Commencement of therapy was under consideration when, 3 months from first isolation of M.abscessus, paraesthesia developed in her hands and feet with associated small joint arthropathy. A subtle purpuric rash was evident on the lower limbs. Nerve conduction studies confirmed an axonal loss sensorimotor neuropathy. Erythrocyte Sedimentation Ratio was elevated at $49 \mathrm{~mm} / \mathrm{hr}$, Perinuclear Anti-Neutrophil Cytoplasmic Antibodies (P-ANCA) titre was highly positive at $80 \mathrm{AI}$ and MyeloperoxidaseANCA (MPO-ANCA) was > 8 AI with Proteinase-3ANCA (PR3-ANCA) $<0.2$ AI. Renal function and urine microscopy were normal. A diagnosis of microscopic polyangiitis was made. Treatment with Cyclophosphamide and high dose Prednisolone was commenced with full resolution of her paraesthesia.

Within 4 months of first isolation, all sputum samples $(n=4)$ were culture positive and $66 \%$ of those samples were smear positive for $M$. abscessus. C-Reactive Protein was elevated at $86.2 \mathrm{mg} / \mathrm{l}$. A repeat CT thorax showed extensive bronchiectasis with underlying collapse in the right upper and lower lobes. There was extensive tree-in-bud change within the right lower lobe, scattered pulmonary nodules and small areas of ground glass shadowing in both lungs. Deteriorating radiological appearances and commencement of active immunosuppression prompted urgent initiation of $M$. abscessus treatment.

\section{Treatment}

Induction phase treatment comprised intravenous (IV) Cefoxitin and Amikacin with oral Clarithromycin and Minocycline and was tolerated for 2 weeks. Long-term nebulised Amikacin was commenced, along with oral Moxifloxacin, Minocycline and Clarithromycin.

A month later she attended the Emergency Department (ED) with rupture of her right Achilles tendon secondary to Moxifloxacin. This was stopped immediately and replaced with Linezolid. Despite dose reduction this was discontinued due to intolerable nausea, diarrhoea and angular stomatitis. She remained on oral Minocycline, Clarithromycin and nebulised Amikacin. Cyclophosphamide was changed to Azathioprine as long-term immunosuppression, but this was poorly tolerated due 

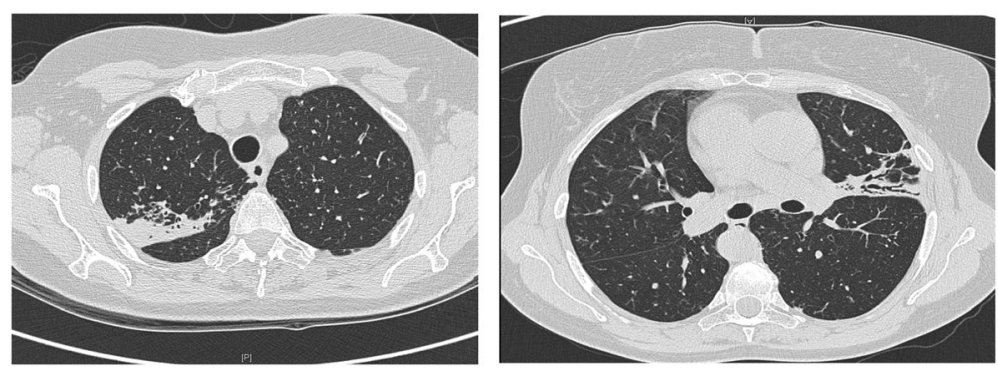

Fig. 1 CT Thorax 2007: CT Thorax from 2007 demonstrating bi-apical scarring, right middle lobe atelectasis, right upper lobe cylindrical bronchiectasis and reticulo-nodular densities in both lower lobes

to nausea. Significant alopecia then developed; attributed to Minocycline, which was also discontinued. Nebulised Meropenem was therefore added to maintain triple antibiotic therapy.

On review 17 months after initiation of treatment her pulmonary disease appeared more stable. A regime of nebulised Meropenem, nebulised Amikacin and oral Clarithromycin was tolerated. Smear and culture negativity was maintained for a further 12 months on this regime. P-ANCA and MPO-ANCA titres remained within normal limits during this period.

Twenty months after starting treatment, cultures again became positive for M.abscessus. A month after culture positivity returned, she presented to the ED with visual loss secondary to a right branch retinal vein occlusion. MPO-ANCA had again become highly positive at $>8$ AI and P-ANCA titre was 160 AI. Due to active vasculitis with impending visual loss from associated hypercoagulability and thromboembolism higher dose immunosuppression was required. She commenced Rituximab and Methylprednisolone infusions weekly for 4 weeks, followed by 6 monthly Rituximab. Clinical response was achieved with CD19 count reducing from $46 \%$ to $0 \%$. Within the following 12 months sputum samples became smear positive again. High resolution CT chest demonstrated extensive airspace opacification, ill-defined nodularity and tree in bud change. (Fig. 2)

\section{Outcome and follow-up}

Further assessment of immunological function was carried out, including vaccination response, respiratory oxidative burst, Mannan Binding Lectin, IgG subsets, complement levels, alternative and innate signalling pathways. Specific testing of interferon pathways demonstrated a very low interferon-gamma (IFN- $\gamma$ ) level, with low production of Interleukin-17 (IL-17) but no autoantibodies to IFN- $\gamma$.

No other organisms, including S.aureus have been detected by standard sputum culture since 2012. Four years from first isolation she remained M.abscessus smear and culture positive. Clarithromycin resistance was now detectable necessitating its cessation. A further 3 weeks of IV Amikacin, Cefoxitin and Tigecycline was commenced. Long-term treatment continued with nebulised Meropenem and Amikacin.

A third oral agent would optimise long-term therapy but intolerances, resistance and risk of visual loss prevent the use of almost all suitable oral agents. Clofazimine was not commenced due to risk of further visual loss. A trial of oral Co-trimoxazole [5] resulted in intolerable nausea and had to be stopped. As no third oral agent could be used, pulsed IV antibiotics have been introduced. The optimal duration between IV courses is unknown and is currently based on recurrence of smear positivity or deteriorating symptoms. The first repeat IV antibiotic course maintained smear negativity for
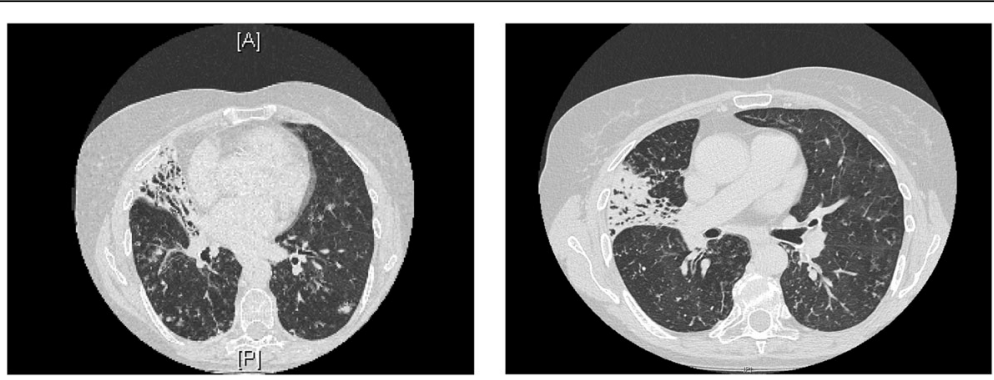

Fig. 2 CT Thorax 2016: High resolution CT Chest from 2016 demonstrating extensive airspace opacification, ill-defined nodularity and tree in bud change 
6 months. Recurrence of smear positivity and associated clinical deterioration have required further IV courses (Table 1).

\section{Discussion}

This case highlights a rare systemic complication of NTM-PD and is the first reported case of ANCA-positive vasculitis as a consequence of $M$. abscessus infection. It demonstrates the complexity of managing NTM-PD including therapeutic challenges and the relationship between immune function and NTM infection.

NTM-PD is increasing in incidence and prevalence. Determining the clinical relevance of NTM isolates can be difficult [2]. M. abscessus is the 3rd most commonly isolated NTM in the USA [5]. It predominantly affects white, female non-smokers over the age of 60 [5]. It can occur in the absence of underlying lung disease where the course can be slow and indolent. In the setting of pre-existing lung disease such as CF or bronchiectasis, it can be fulminant and rapidly progressive [5]. The radiographic picture is of inflammatory nodules, tree-in-bud opacities and cavitation particularly in areas of underlying bronchiectasis [4].

There is wide variation in how patients with $M$. abscessus NTM-PD are treated [4]. An intensive induction phase consisting of three IV antibiotics administered for several weeks in combination with an oral agent is common $[4,5]$. This is usually followed by at least two oral antimicrobials in addition to a macrolide antibiotic and inhaled antibiotic(s) [4]. Side effects of antimicrobial therapy are significant and can limit treatment options over a prolonged treatment period. In one study $65 \%$ patients stopped at least one antibiotic due to adverse events or toxicity [6]. In this case the additive toxicity of both anti-microbial and immunosuppressive agents further increased the side effect profile (Table 2).

The association between vasculitis and chronic suppurative lung conditions $[7,8]$ is thought to result from excessive humoral immune responses secondary to circulating immune complexes [8]. Pulmonary infection may trigger vasculitis through induction of ANCA antigen expression on the surface of neutrophils [9]. The temporal relationship between M.abscessus growth, ANCA positivity and vasculitic symptoms reduces the chance of the two being unrelated, suggesting M.abscessus was a causative trigger for the vasculitides. S.aureus had been chronically cultured since presentation with no prior evidence of vasculitis reducing the likelihood of S.aureus being a causative factor. Previous case reports have described ANCA-positive vasculitis secondary to M.avium complex [10]. Leukocytoclastic vasculitis linked to Salmonella has been reported in children with genetic Interferon-gamma deficiency [11]. Leukocytoclastic vasculitis in an individual with interferon-gamma autoantibodies and disseminated $M$. abscessus has also been reported [12]. Increased susceptibility to mycobacterial disease has been reported in genetic interferon defects but a causal link with associated vasculitides has not been established [13]. It is possible the reduction in IFN- $\gamma$ seen in this case may have contributed both to development of vasculitis and poor treatment response.

The use of IFN- $\gamma$ had been considered in this case. IFN- $\gamma$ production is integral to mycobacterial defence through activation of both innate and adaptive immune systems via the interleukin12-IFN- $\gamma$ pathway [13, 14]. IFN- $\gamma$ facilitates mycobacterial killing by enhancing phagocytosis and expression of oxygen free radicals [14]. Administration is associated with a flu like syndrome [15-17]. Intramuscular IFN- $\gamma$ as adjuvant therapy for $M$. avium complex resulted in clinical benefit over antibiotics alone $[5,15]$ but of insufficient magnitude to warrant recommending routine use [5]. IFN- $\gamma$ therapy in management of $M$. abscessus disease is limited to case reports of use in disseminated disease [16, 18].

Rituximab has been successfully used in disseminated $M$. abscessus disease refractory to antibacterial chemotherapy in those with IFN- $\gamma$ autoantibodies [12, 19]. It can restore IFN- $\gamma$ signalling in these individuals $[12,19]$. Whether this is true in those without autoantibodies, as in this case is unknown, as is any potential interaction between Rituximab and IFN- $\gamma$ therapy. In this case monitoring Rituximab response first was considered of lowest risk but the addition of IFN- $\gamma$ could be re-visited.

Treatment regimens for systemic vasculitis comprise high dose induction immunosuppression followed by oral maintenance therapy for 12-18 months [9]. The challenge in this case was balancing adequate immunosuppression with sufficient antimicrobial therapy to maintain clinical stability alongside smear and culture negativity. Reductions in immunosuppression triggered vasculitic flares with sight threatening consequences whilst increases in immunosuppression led to a deterioration in NTM-PD.

This case highlights the complex interplay between immune function and NTM-PD both as a complication and a cause of disease $[13,14,20]$. Defects in IFN- $\gamma$ pathways, both acquired and genetic are associated with NTM-PD [14, 20]; as are defects in macrophage and dendritic cell function and cytokine signalling [5, 13]. Whole genome sequencing has demonstrated higher rates of genetic variants in immune, Cystic Fibrosis Transmembrane Conductance Regulator, cilial function and connective tissue categories in people with NTM-PD. [21] Screening for immune defects in those with NTM-PD who fail to respond to "standard" treatment is increasingly recommended [5] and may allow better prognostication and management in the future. Equally, those with known immune dysfunction should be screened for NTM. 


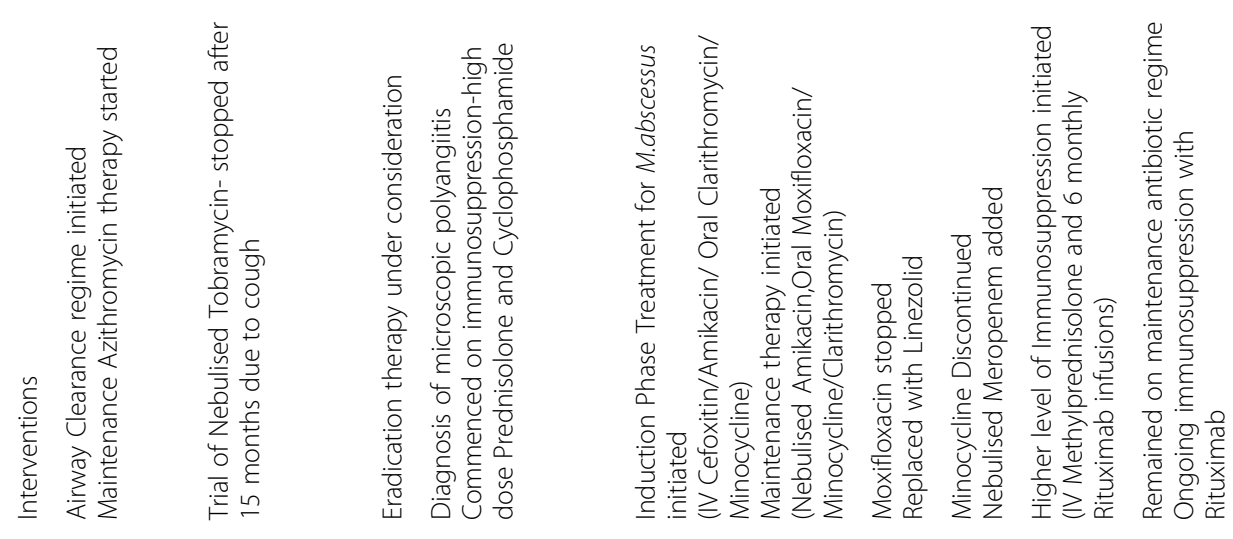

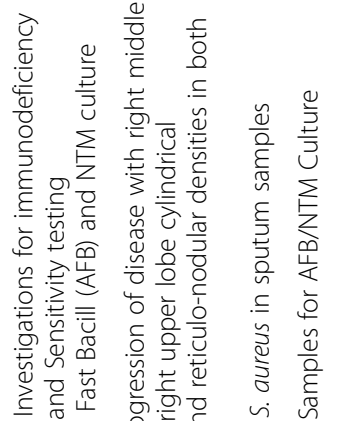

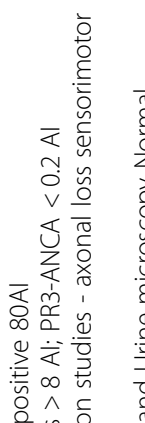

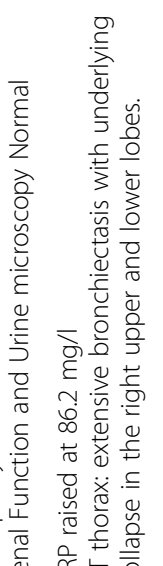

完

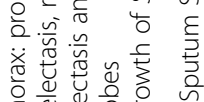

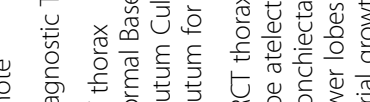

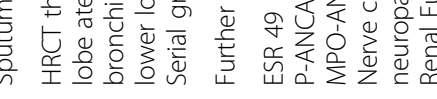

준 $\overline{\overline{0}}$

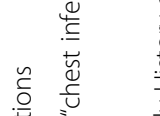

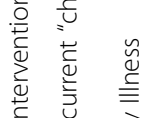

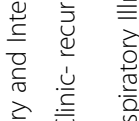

产

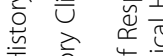

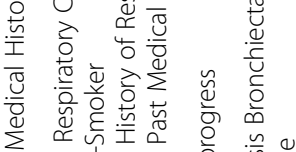

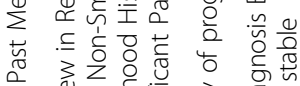

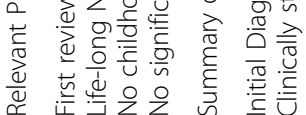
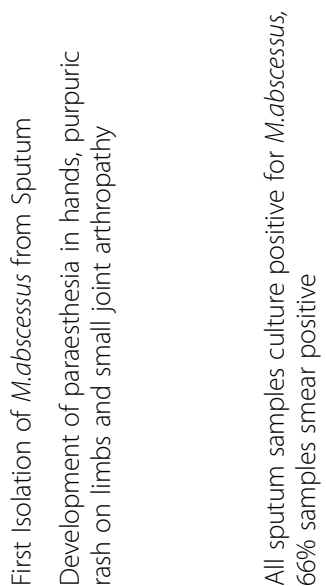

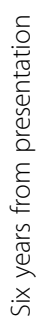

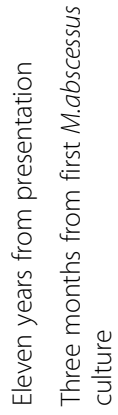

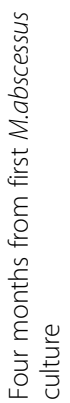
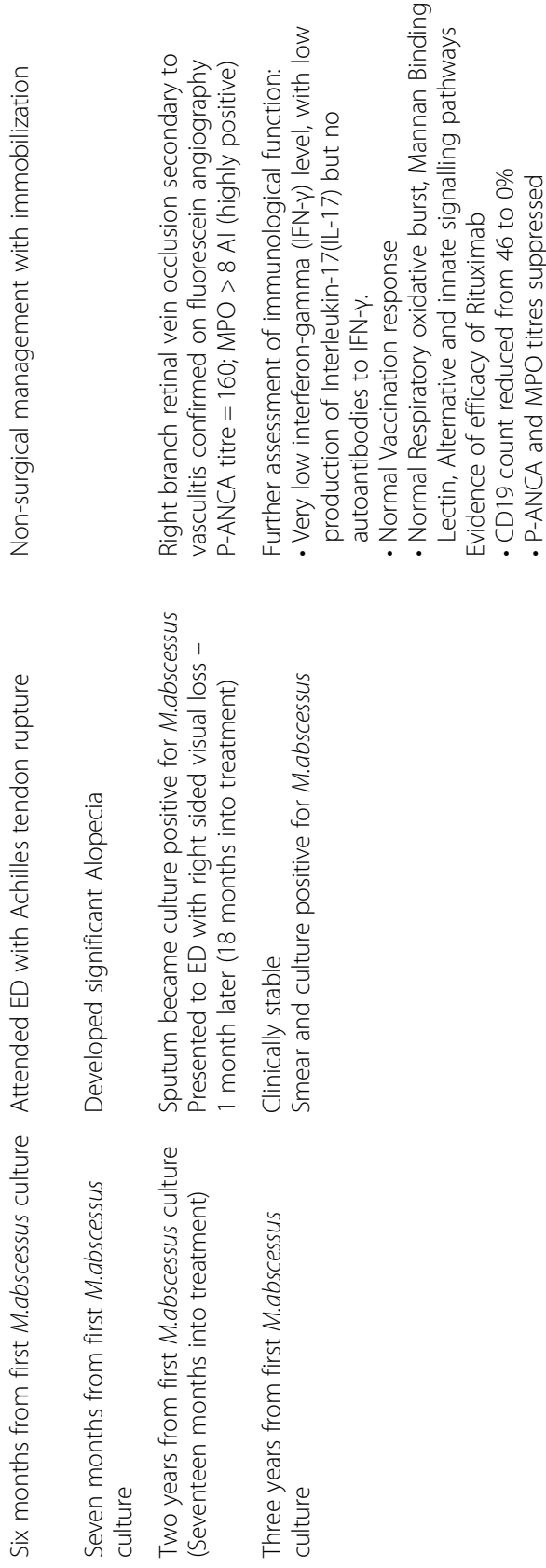


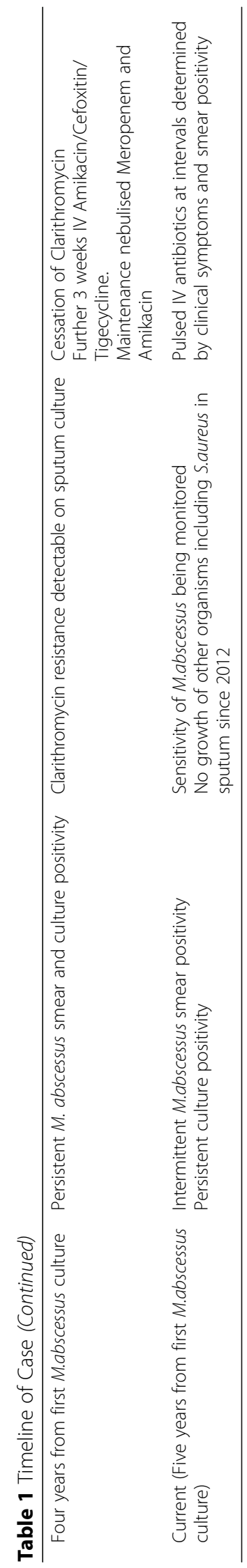


Table 2 Adaption from RA Floto, KN Olivier, L. Saiman et al.US Cystic Fibrosis Foundation and European Cystic Fibrosis Society consensus recommendations for the management of non-tuberculous mycobacteria in individuals with cystic fibrosis. Thorax; 2016:71:i1-i22; Haworth CS, Banks J, Capstick T et al. British Thoracic Society Guidelines for the Diagnosis and Management of Non-Tuberculous Mycobacterial Pulmonary Disease (NTM-PD). Thorax; 2017;72:2; Lallana EC and Fadul CE. Toxicities of Immunosuppressive Treatment of Autoimmune Neurologic Diseases. Curr Neuropharmacol. 2011 Sep; 9 (3):468-477 [24]

\begin{tabular}{|c|c|}
\hline Drug & Common Side Effects \\
\hline Amikacin & $\begin{array}{l}\text { Nephrotoxicity, } \\
\text { Ototoxicity: irreversible vestibulocochlear nerve damage. }\end{array}$ \\
\hline Azithromycin & $\begin{array}{l}\text { Nausea, Vomiting, Diarrhoea, Fulminant Hepatitis, Pseudomembranous Colitis } \\
\text { Ototoxicity } \\
\text { Prolonged QT }\end{array}$ \\
\hline Clarithromycin & $\begin{array}{l}\text { Hepatitis } \\
\text { Taste Disturbance } \\
\text { Prolonged QT }\end{array}$ \\
\hline Cefoxitin & $\begin{array}{l}\text { Fever, Rash } \\
\text { Eosinophilia, Anaemia, Leucopenia, Thrombocytopenia }\end{array}$ \\
\hline Clofazimine & $\begin{array}{l}\text { Pink Brownish-Black Discoloration of Skin, Ichthyosis and dry skin } \\
\text { Enteropathy, Nausea and Vomiting } \\
\text { Conjunctival pigmentation, Dimness of vision and dry eyes. }\end{array}$ \\
\hline Co-Trimoxazole & $\begin{array}{l}\text { Nausea, Vomiting, Diarrhoea } \\
\text { Anaemia, Leucopenia, Thrombocytopenia } \\
\text { Hyponatraemia, Hyperkalaemia } \\
\text { Fever, Rash, Steven Johnson Syndrome }\end{array}$ \\
\hline Ethambutol & $\begin{array}{l}\text { Optic Neuritis } \\
\text { Hyperuricaemia } \\
\text { Peripheral Neuropathy }\end{array}$ \\
\hline Imipenem & $\begin{array}{l}\text { Rash and Urticaria } \\
\text { Hepatitis, Nausea, Vomiting Diarrhoea }\end{array}$ \\
\hline Linezolid & $\begin{array}{l}\text { Anaemia, Leucopenia, Thrombocytopenia } \\
\text { Peripheral Neuropathy } \\
\text { Optic Neuritis }\end{array}$ \\
\hline Moxifloxacin & $\begin{array}{l}\text { Nausea, Vomiting, Diarrhoea } \\
\text { Insomnia, Agitation, Anxiety } \\
\text { Tendonitis } \\
\text { Photosensitivity } \\
\text { Prolonged QT }\end{array}$ \\
\hline Minocycline & $\begin{array}{l}\text { Photosensitivity } \\
\text { Nausea, Vomiting, Diarrhoea, Dysphagia } \\
\text { Vertigo, Headache } \\
\text { Skin Hyperpigmentation }\end{array}$ \\
\hline Rifampicin & $\begin{array}{l}\text { Orange discoloration of bodily fluids } \\
\text { Hepatitis, Nausea, Vomiting, Diarrhoea } \\
\text { Fever, Chills } \\
\text { Thrombocytopenia } \\
\text { Renal Failure } \\
\text { Increased hepatic metabolism of numerous drugs }\end{array}$ \\
\hline Streptomycin & $\begin{array}{l}\text { Nephrotoxicity } \\
\text { Ototoxicity }\end{array}$ \\
\hline Tigecycline & $\begin{array}{l}\text { Nausea, Vomiting, Diarrhoea, Pancreatitis, Bilirubinaemia } \\
\text { Increased risk of infections: sepsis/septic shock } \\
\text { Hypoproteinaemia }\end{array}$ \\
\hline Corticosteroids & $\begin{array}{l}\text { Cataract } \\
\text { Increased risk of infection } \\
\text { Type } 2 \text { Diabetes Mellitus } \\
\text { Osteoporosis } \\
\text { Gastrointestinal bleeding } \\
\text { Altered Mood - rarely psychosis } \\
\text { Proximal myopathy } \\
\text { Hypertension }\end{array}$ \\
\hline Cyclophosphamide & $\begin{array}{l}\text { Leucopenia } \\
\text { Haemorrhagic Cystitis }\end{array}$ \\
\hline
\end{tabular}


Table 2 Adaption from RA Floto, KN Olivier, L. Saiman et al.US Cystic Fibrosis Foundation and European Cystic Fibrosis Society consensus recommendations for the management of non-tuberculous mycobacteria in individuals with cystic fibrosis. Thorax; 2016:71:i1-i22; Haworth CS, Banks J, Capstick T et al. British Thoracic Society Guidelines for the Diagnosis and Management of Non-Tuberculous Mycobacterial Pulmonary Disease (NTM-PD). Thorax; 2017;72:2; Lallana EC and Fadul CE. Toxicities of Immunosuppressive Treatment of Autoimmune Neurologic Diseases. Curr Neuropharmacol. 2011 Sep; 9 (3):468-477 [24] (Continued)

\begin{tabular}{ll}
\hline Drug & Common Side Effects \\
\hline & Infertility \\
& Long term increase risk of cancer e.g. Bladder Malignancy, Myeloproliferative disorders \\
Azathioprine & Nausea, Hair Loss, Skin Irritation \\
& Hepatotoxicity, Pancreatitis \\
& Leucopenia \\
Rituximab & Fatigue, Hair loss Diarrhoea \\
& Increased risk of malignancy \\
& Rash \\
& Gl upset \\
\hline
\end{tabular}

Striking the balance between managing infection and immunosuppression is an increasing issue in clinical medicine with rising numbers of patients receiving biologics, immunosuppressive agents and increasing lifespan post haematological and solid organ transplants [5, $22,23]$. Oral corticosteroid use is up to eight times higher amongst cases of NTM infection [22]. Rates of NTM-PD are five to ten fold higher in patients on anti-TNF alpha therapies [23]. Whilst other biological agents, carry a theoretical increased risk of NTM there is little data on infection rates [15]. Acquisition of NTM infection is likely due to disruption or depletion of cell mediated immunity which is a critical component of host defence against mycobacterium [14]. Adequate

Table 3 Key Learning points and Patient Perspective

\footnotetext{
Patient Perspective

"When I was diagnosed with bronchiectasis in my late forties, it did not affect me day to day and I continued to work, raise my family and have an active social life. However, since being diagnosed with

Mycobacterium Abscessus and Vasculitis, I have struggled with tiredness and progressive shortness of breath especially when exerting myself. I am no longer as active as I wish to be, in particular I struggle to keep up with my toddler grand-daughter and make it around the golf course. However, I make the effort to continue to take light exercise every day by meeting friends for coffee, going shopping and short walks when the weather permits."

Learning Points

- NTM Pulmonary disease is increasing in prevalence and should be screened for in in at risk groups. NTM-PD should also be considered if appropriate radiological appearances and /or symptoms develop in these groups.

- Treatment regimens are toxic and difficult to tolerate and therefore timing of treatment and goals of treatment are important as well as clear communication with patients in this regard

- With increases in therapies which impair immunity the rates of NTM-PD in these groups may continue to rise

- Vasculitis triggered by NTM is a rare but significant complication of NTM and this is the first reported case of this being due to M.Abcessus. - Balancing effective immunosuppression with active treatment for infection is challenging and requires specialist expertise and collaborative working between specialist services.
}

screening of high-risk populations for NTM is necessary to further understanding of disease rates and facilitate appropriate intervention $[1,5,22]$ (Table 3).

\section{Conclusion}

Effective treatment of NTM-PD requires effective communication and team working between specialities treating this complex group of patients. Balancing immunosuppressive with anti-microbial regimes and their relative toxicities is challenging and requires frequent assessment, monitoring and treatment adjustment. Further research will refine management approaches and improve our understanding of the role of innate, adaptive and auto-immune dysfunction and the incidence of related complications including vasculitis.

\section{Abbreviations}

AFB: Acid Fast Bacilli; ANCA: Anti-Neutrophil Cytoplasmic Antibodies; CT: Computed Tomography; ED: Emergency Department; IFN- $\gamma$ : Interferon - gamma; IL-17: Interleukin-17; IV: Intra-venous; M.a.abscessus: Mycobacterium abscessus abscessus; M.a.bolletii: Mycobacterium abscessus bolletii; M.a.massiliense: Mycobacterium abscessus massiliense; M.abscessus: Mycobacterium abscessus; MPO-ANCA: Myeloperoxidase Anti-Neutrophil Cytoplasmic Antibodies; NTM: Non-Tuberculous Mycobacteria; NTM-PD: Non-

Tuberculous Mycobacterial Pulmonary disease; P-ANCA: Perinuclear Anti-Neutrophil Cytoplasmic Antibodies; PR3-ANCA: Proteinase - 3Anti-Neutrophil Cytoplasmic Antibodies; S.Aureus: Staphyloccocus aureus; TNF-alpha: Tumour Necrosis Factor - alpha

\section{Acknowledgements}

Not applicable.

Funding

Not applicable.

Availability of data and materials Not applicable.

Authors' contributions

$\mathrm{CA}$ and GD were major contributors to writing and creating the manuscript. AJ, GW, SC and DGD contributed to writing and editing the manuscript. All authors read and approved the final manuscript. 


\section{Authors' information}

The authors represent the complexity in managing this case across multiple hospital sites and specialities. Respiratory speciality input has been provided both at Belfast City Hospital, Belfast (CA, GD, SC and DGD) and at Royal Brompton and Harefield Hospital London (AJ) with Rheumatology specialist input provided at Musgrave Hospital, Belfast (GW).

\section{Ethics approval and consent to participate} Not Applicable.

\section{Consent for publication}

Written informed consent for publication of their clinical details and/or clinical images was obtained from the patient. A copy of the consent form is available for review by the Editor of this journal.

\section{Competing interests}

The authors declare that they have no competing interests.

\section{Publisher's Note}

Springer Nature remains neutral with regard to jurisdictional claims in published maps and institutional affiliations.

\section{Author details}

'Centre for Experimental Medicine, Queen's University Belfast, 97 Lisburn Road, Belfast BT9 7BL, Northern Ireland. ${ }^{2}$ Regional Respiratory Centre, Belfast City Hospital, 51 Lisburn Road, Belfast BT9 7AB, Northern Ireland. ${ }^{3}$ Department of Respiratory Medicine, Royal Brompton Hospitals, Sydney Street, London SW3 6NP, England. ${ }^{4}$ Department of Rheumatology, Musgrave Park Hospital, Stockmans Ln, Belfast BT9 7JB, Northern Ireland.

Received: 2 May 2018 Accepted: 29 October 2018

Published online: 19 November 2018

\section{References}

1. Bonaiti G, Pesci A, Marruchella A, et al. Nontuberculous Mycobacteria in Noncystic Fibrosis Bronchiectasis. BioMed Res Int. 2015;2015:197950. https://doi.org/10.1155/2015/197950.

2. Moore J, Kruijsharr M, Ormerod LP, et al. Increasing reports on nontuberculous mycobacteria in England, Wales and Northern Ireland, 19952006. BMC Public Health. 2010;10:612

3. Griffith DE, Aksamit T, Brown-Elliott BA, et al. An official ATS/IDSA statement: diagnosis, treatment, and prevention of nontuberculous mycobacterial diseases. Am J Respir Crit Care Med. 2007;175(4):367-416.

4. Floto RA, Olivier KN, L. Saiman et al.US Cystic Fibrosis Foundation and European cystic fibrosis society consensus recommendations for the management of nontuberculous mycobacteria in individuals with cystic fibrosis. Thorax. 2016;71:11-i22.

5. Haworth CS, Banks J, Capstick T, et al. British Thoracic Society Guidelines for the Diagnosis and Management of Non-Tuberculous Mycobacterial Pulmonary Disease (NTM-PD). Thorax. 2017;72(Suppl.2).

6. Jarand J, Levin A, Zhang $L$, et al. Clinical and microbiologic outcomes in patients receiving treatment for Mycobacterium abscessus pulmonary disease. Clin Infect Dis. 2011;52:565-71.

7. Tanaka E, Tada K, Amitani R, et al. Systemic hypersensitivity Vasculitis associated bronchiectasis. Chest. 1992;102(2):647-9.

8. Bruce IN, McAteer JA, Gardiner PV, et al. Chronic supprative lung disease with associated vasculitis. Postgrad Med J. 1995;71:24-7.

9. Tagboto SK, Venkatesh AG. Progressive dyspnoea following the treatment of Mycobacterium abscessus infection in an individual with relapsing granulomatosis with polyangiitis (Wegener's), complicated by hearing loss requiring cochlear implantation. BMC Pulm Med. 2012;12:47.

10. Asano S, Mizuno S, Okachiet S, et al. Antineutrophil cytoplasmic antibodyassociated Vasculitis superimposed on infection-related glomerulonephritis secondary to pulmonary Mycobacterium avium complex infection. Intern Med. 2016;55:2439-45.

11. Filiz S, Kocacik Uygun DF, Verhard EM, et al. Cutaneous Leukocytoclastic Vasculitis due to Salmonella enteritidis in a child with Interleukin-12 receptor Beta-1 deficiency. Paediatr Dermatol. 2014;31(2):236-40.

12. Czaja CA, Merkel PA, Chan ED, et al. Rituximab as Successful Adjunct Treatment in a Patient With Disseminated Nontuberculous Mycobacterial Infection Due to Acquired Anti-Interferon- $\gamma$ Autoantibody. Clin Infect Dis. 2014;58(6):115-8.
13. Wu U-I, Holland SM. Host susceptibility to non-tuberculous mycobacterial infections. Lancet Infect Dis. 2015:15:968-80.

14. Haverkamp MH, Van Diesel JT, Holland SM. Human host genetic factors in nontuberculous mycobacterial infection; lessons from single gene disorders affecting innate and adaptive immunity and lessons from molecular defects in interferon- $\gamma$-dependent signalling. Microbes Infect. 2006:8:1157-66.

15. Milanés-Virelles MT, García-García I, Santos-Herrera Y, et al. Adjuvant interferon gamma in patients with pulmonary atypical Mycobacteriosis: a randomized, double blind,placebo-controlled study. BMC Infect Dis. 2008;8:17.

16. Colsky A, Hanly A, Elgart G, Kerdel F. Treatment of Refractory Disseminated Mycobacterium abscessus Infection With Interferon Gamma Therapy. Arch Dermatol. 1999;135(2):125-7.

17. Gallin JI, Farber JM, Holland SM. Interferon-y in the Management of Infectious Diseases. Ann Intern Med. 1995;123:216-24.

18. Rivera R, Alrabaa S, List A, Greene J. Successful Treatment of Refractory Disseminated Mycobacterium abscessus Infection Using Interferon Gamma. Infect Dis Clin Prac. 2011;19(2):142-5.

19. Browne SK, Zaman R, Sampaio E, et al. Anti-CD20 (rituximab) therapy for anti-IFN autoantibody-associated nontuberculous mycobacterial infection. Blood. 2012;119(17):3933-9.

20. Lim A, Allison C, Price P, et al. Susceptibility to pulmonary disease due to mycobacterial avium-intracellulare complex may reflect low IL-17 and high IL-10 responses rather than TH1 deficiency. Clin Immunol. 2017;137:296-320.

21. Szymanski E, Leung JM, Fowler CJ, et al. Pulmonary nontuberculous mycobacterial infection: a multisystem, Multigenic Disease. Am J Respir Crit Care Med. 2015:192(5):618-28.

22. Henkle E, Winthrop K. Nontubercuolous mycobacteria infections in immunocompromised hosts. Clin Chest Med. 2015;36(1):91-9.

23. Winthrop KL, Baxter R, Liu L. Mycobacterial diseases and anti-tumour necrosis factor therapy in USA. Ann Rheum Dis. 2013;72:37-42.

24. Lallana EC, Fadul CE. Toxicities of immunosuppressive treatment of autoimmune neurologic diseases. Curr Neuropharmacol. 2011;9(3):468-77.

\section{Ready to submit your research? Choose BMC and benefit from:}

- fast, convenient online submission

- thorough peer review by experienced researchers in your field

- rapid publication on acceptance

- support for research data, including large and complex data types

- gold Open Access which fosters wider collaboration and increased citations

- maximum visibility for your research: over $100 \mathrm{M}$ website views per year

At $\mathrm{BMC}$, research is always in progress.

Learn more biomedcentral.com/submission 